\title{
Estudo das interações medicamentosas em idosos residentes em instituição de longa permanência
}

\author{
Study of drug interactions in elderly \\ residents in institution for long-stay
}

Elisangela GERBER*, Adriana de Oliveira CHRISTOFF Curso de Farmácia Faculdade Integrada do Brasil - UNIBRASIL, Rua: Konrad Adenauer,442 - Tarumã,Curitiba(PR),Brasil.E-mail: gerberelis@hotmail.com

\begin{abstract}
Physiological changes related to aging imply to an increased consumption of drugs, wich makes it subject to the practice of polypharmacy an drug interactions consequently. Some interactions may, jeopardize the safety and effectiveness of treatment, increasing the pharmacologic effects causing toxicity, accentuate side effects, develop an adverse reaction to medication and even increase morbidity and mortality. Thus, the objective of this work was a study of the major drug interactions by evaluation the medical prescriptions the three elderly individuals residing in long-stay institution for a period of 12 months . The three elderly selected subjects used eight to ten medications daily. We have found 17 possible drug interactions classified interactions pharmacokinetic involving the internal mechanisms of absorption, distribution an transformation of drugs and biotransformation of drugs and pharmacodynamic, interactions through mechanisms of potentiation, antagonism and summation. Compared to the results, it was suggested corrective measures to provide safety, therapeutic efficacy and quality of life among the elderly.
\end{abstract}

KEYWORDS: aging; drug interactions; pharmaceutical care.

\section{RESUMO}

Mudanças fisiológicas relacionadas à senescência levam o idoso a um consumo aumentado de medicamentos, o que o torna sujeito à prática da polifarmácia e, consequentemente, as interações medicamentosas. Algumas interações podem comprometer a segurança e efetividade do tratamento, aumentar os efeitos farmacológicos causando toxicidade, acentuar os efeitos colaterais, desenvolver uma reação adversa ao medicamento e até elevar a morbimortalidade. Desta forma, o objetivo deste trabalho foi realizar um estudo das principais interações medicamentosas através da avaliação de prescrições médicas de três indivíduos idosos residentes em instituição de longa permanência por um período de 12 meses. Os três idosos selecionados utilizavam de oito a dez medicamentos diariamente. Foram encontradas 17 possíveis interações medicamentosas, classificadas em interações farmacocinéticas, envolvendo mecanismos de absorção, distribuição e biotransformação dos fármacos e interações farmacodinâmicas por mecanismos de potencialização, antagonismo e somação. Frente aos resultados obtidos, sugeriu-se medidas corretivas a fim de proporcionar segurança, eficácia terapêutica e qualidade de vida ao idoso.

PALAVRAS CHAVE: Atenção Farmacêutica; Idoso; Interações Medicamentosas. 


\section{LISTA DE ABREVIAÇÕES}

AF atenção farmacêutica

BZD benzodiazepínicos

CYP enzimas do citocromo P450, são agrupados em famílias e subfamílias

D2 receptor da dopamina que se liga a proteína Gi para inibir adenililciclase.

DPOC doença pulmonar obstrutiva crônica

ECA enzima conversora de angiotensina

GABAA receptor ionotrópico subtipo A do ácido gama-aminobutírico

HMGCOA redutase - 3-hidroxi-3-metilglutaril coenzima-a redutase

ICC insuficiência cardíaca congestiva

IM interações medicamentosas

QT intervalo QT corresponde ao tempo que decorre desde o princípio da despolarização repolarização dos ventrículos

RAM reação adversa ao medicamento

SNC sistema nervoso central

$\boldsymbol{\alpha 1}$ e $\boldsymbol{\alpha 2}$ receptores alfa-adrenérgicos

$\boldsymbol{\beta 1}$ e $\boldsymbol{\beta 2}$ receptores beta- adrenérgicos

5HT2A subtipo de receptor da 5-hidroxitriptamina (serotonina)

$\boldsymbol{\mu}$ receptor opióide

\section{INTRODUÇÃO}

O crescimento da população de idosos pode ser evidenciado em todo Brasil e atribui-se a este fenômeno a queda das taxas de fecundidade, da redução da mortalidade e do aumento da expectativa de vida (1).

O envelhecimento é acompanhado por um processo de alterações biológicas, morfológicas, funcionais e psicológicas progressivas, elevando a frequência de doenças crônico-degenerativas que acometem os idosos $(2,3)$.

Ao conviverem mais com problemas crônicos de saúde, os idosos estão sujeitos a uma alta demanda de medicamentos. O uso de múltiplos fármacos leva a prática da polifarmácia (3). A polifarmácia, conceituada como uso concomitante de vários fármacos (4), favorece a ocorrência de interações medicamentosas. Uma interação ocorre quando um medicamento influencia a ação de outro, podendo ser benéfica quando a associação de fármacos promovem efeitos semelhantes proporcionando uma boa eficácia terapêutica ou redução dos efeitos adversos, entretanto uma interação maléfica pode diminuir a eficácia terapêutica, aumentar excessivamente os efeitos farmacológicos causando toxicidade, acentuar os efeitos colaterais, desenvolver uma reação adversa ao medicamento (RAM) e até elevar a morbimortalidade $(4,5)$.
As interações medicamentosas (IM) podem ser classificadas, conforme os mecanismos envolvidos, em interação farmacocinética ou farmacodinâmica. A primeira ocorre quando um medicamento altera a absorção; distribuição; metabolismo ou excreção de outros medicamentos, enquanto a outra resulta da capacidade de um medicamento interagir com o sitio de ação, afetando o mecanismo de ação de outros fármacos (4).

Os idosos tornam-se mais vulneráveis às IM, visto que nesta etapa da vida o metabolismo hepático, homeostático e renal estão sujeitos ao comprometimento, logo, principalmente a distribuição e metabolização das drogas podem ficar prejudicadas e os efeitos adversos intensificados. Alguns exemplos de efeitos aumentados nos idosos são: hipotensão postural com fármacos que diminuem a pressão sanguínea; desidratação, hipovolemia e distúrbios eletrolícos com diuréticos; e hemorragias com anticoagulantes orais $(5,6,7)$.

Portanto o serviço de Atenção Farmacêutica (AF) é essencial para o monitoramento da associação dos medicamentos, através da avaliação da prescrição e da identificação de situações que levam as IM e eventos adversos, possibilitando a promoção de ações preventivas (8). Atenção Farmacêutica é o exercício do profissional farmacêutico a favor do paciente com o objetivo de alcançar o resultado terapêutico desejado e que melhore a qualidade de vida do mesmo $(9,10)$.

A avaliação farmacêutica da prescrição não visa intervir nas atribuições do médico, mas proporcionar uma farmacoterapia racional, segura e custo-efetiva, envolvendo aspectos de orientação e atendimento farmacêutico, dispensação e acompanhamento/ seguimento farmacêutico (11).

Frente ao exposto, a presente pesquisa teve como objetivo realizar um estudo das interações medicamentosas em 3 idosos institucionalizados identificando a presença ou não de possíveis interações entre os medicamentos prescritos, com a finalidade de propor medidas corretivas para reduzir os efeitos adversos e colaterais advindos das interações.

\section{MATERIAIS E MÉTODOS}

O presente trabalho foi aprovado pelo Comitê de Ética em Pesquisa das Faculdades Integradas do Brasil - UNIBRASIL, sob o número 36/2010, de acordo com as exigências das Resoluções Nacionais 196/96 e demais relacionadas a pesquisas envolvendo seres humanos.

Trata-se de um projeto de extensão, desenvolvido em uma Instituição de Longa Permanência na cidade de Curitiba-Paraná com uma população total de 96 idosos do sexo masculino, porém far-se-á um recorte com (n) amostral de três idosos. A pesquisa seguiu modelo ob- 
servacional, prospectivo e foi constituída de duas fases: primeiro realizou-se uma investigação documental das prescrições médicas de fármacos de uso contínuo e relatórios de evolução médica e da enfermagem de três idosos, do sexo masculino, com mais de 60 anos, que utilizavam cinco ou mais medicamentos por dia, durante o período de fevereiro de 2010 a fevereiro de 2011. Em seguida realizou-se a fase de estudo, por meio de revisões bibliográficas, utilizando bases de dados nacionais e internacionais a fim de identificar possíveis interações medicamentosas e eventos adversos.

As possíveis interações farmacodinâmicas encontradas foram classificadas como interação por somação, quando a associação dos fármacos promoveu efeitos semelhantes, porém por mecanismo de ação diferente, interação de potencialização, quando o efeito final da associação foi maior que a soma ou adição dos efeitos de cada fármaco e antagonismo entre fármacos de ações contrárias reduzindo a ação de um dos fármacos (12). Quanto às interações farmacocinéticas, evidenciou-se a interferência de fármacos no processo de absorção, distribuição e biotransformação, por mecanismo de inibição do citocromo P450 e eliminação.

\section{RESULTADOS E DISCUSSÃO}

Os idosos foram identificados por letras do alfabeto: Idoso A, B e C, com idade de 66, 71 e 76 anos respectivamente.

Durante a análise das prescrições médicas, verificou-se a presença de oito a dez medicamentos prescritos de uso contínuo diário. Não foram avaliados os medicamentos de uso esporádicos, por representarem um número irrelevante. Alguns destes medicamentos que eram apenas de uso esporádicos passaram a ser de uso contínuo, como o diazepam, clonazepam, paracetamol e paracetamol associado à codeína, portanto, considerados no estudo.

O idoso A, apresenta as seguintes patologias: depressão, doença pulmonar obstrutiva crônica (DPOC), esquizofrenia paranóide e cefaleia intensa. É tabagista, etilista e apresenta crises de abstinência alcoólica. Ao analisar suas prescrições médicas, no período referido anteriormente, constatou-se a possibilidade de ocorrência de 8 IM entre medicamento medicamento e medicamento etanol. Na Tabela 1 encontram-se descritas os horários de administração e as medicações de uso contínuo.

Dentre as principais interações evidenciadas destacam-se as que envolvem os medicamentos de ação sobre o sistema nervoso central (SNC). A coadministração do clonazepam e diazepam, ocasiona uma interação de potencialização. Estes dois fármacos são benzodiazepí- nicos (BZD), portanto são moduladores alostéricos dos receptores GABAA e atuam em sítios de ligação específicos do receptor aumentando a frequência de abertura dos canais de cloreto na presença do GABA endógeno $(13,14,15)$.

Tanto o clonazepam como o diazepam são considerados BZD de ação longa, de alta potência e apresentam uma margem de segurança diminuída quando há associação de dois fármacos hipnóticos / sedativos, consequentemente resulta em um aumento do efeito depressor do SNC, podendo ocasionar uma depressão respiratória $(13,15)$.O centro respiratório é afetado por mudanças na pressão parcial de dióxido de carbono e pressão parcial do oxigênio do sangue, os BZD deprimem levemente a ventilação alveolar e causam acidose respiratória, por promoverem o aumento da pressão de dióxido de carbono o que ocasiona em uma depressão respiratória $(14,15)$.

\section{Tabela 1: Medicamentos de uso contínuo do Idoso A}

\begin{tabular}{|c|c|}
\hline Administração & Medicação \\
\hline $6 \mathrm{~h} 00$ & omeprazol 20mg; paracetamol 500mg \\
\hline \multirow{3}{*}{8 h00 } & clonazepam 0,5 mg; risperidona $2 \mathrm{mg}$; \\
\hline & enalapril 10mg; sertralina 50 mg \\
\hline & diazepam $5 \mathrm{mg}$; ácido fólico $5 \mathrm{mg}$ \\
\hline \multirow{2}{*}{$12 \mathrm{~h} 00$} & ácido acetilsalicílico 100 mg \\
\hline & polivitamínico \\
\hline $14 \mathrm{~h} 00$ & risperidona $2 \mathrm{mg}$ \\
\hline $16 h 00$ & paracetamol 500mg; diazepam $5 \mathrm{mg}$ \\
\hline $20 \mathrm{~h} 00$ & risperidona $4 \mathrm{mg}$; clonazepam $0,5 \mathrm{mg}$ \\
\hline $22 \mathrm{~h} 00$ & paracetamol 500 mg; diazepam 5 mg \\
\hline
\end{tabular}

Fonte: Prescrições médicas do período de fevereiro de 2010 a fevereiro de 2011.

Deve - se considerar que o idoso A tem DPOC e o uso dos BZD, mesmo em doses terapêuticas, comprometem a respiração em portadores desta patologia (15).

Um dos efeitos finais da associação de dois BZD é o prolongamento da sedação, portanto este idoso passará boa parte do dia sonolento, o que poderá ocasionar quedas e fraturas $(15,16)$. Este efeito de sonolência no idoso foi verificado nos períodos da pesquisa.

Outra interação importante de potencialização foi encontrada na administração da risperidona; antipsicótico atípico, antagonista do receptor D2 acoplado à proteína $\mathrm{Gi}$, com o clonazepam e diazepam no mesmo horário, conforme tabela 1, o que acarreta em aumento da depressão do SNC $(4,14,16)$.

A associação do clonazepam com a sertralina evidencia uma interação na biotransformação do clonaze- 
pam por mecanismo de inibição enzimática exercida pela sertralina. As enzimas responsáveis pelo metabolismo dos benzodiazepínicos são: CYP2C19; CYP2D6; CYP3A3/4 e a sertralina inibe as enzimas CYP2C19, CYP2D6 e CYP3A3 o que leva a um aumento dos níveis do clonazepam, potencializando o efeito depressor sobre o SNC $(13,15)$.

$\mathrm{Na}$ associação entre risperidona com sertralina identifica-se uma interação farmacocinética e uma farmacodinâmica. Na interação farmacocinética, a sertralina inibe a CYP2D6 responsável pela metabolização da risperidona, aumentando seus níveis e por consequência, seus efeitos tóxicos $(13,15,18)$. Já a interação farmacodinâmica ocorre por antagonismo competitivo, pois, o mecanismo de ação da sertralina é inibir a receptação da serotonina, deixando o neurotransmissor mais tempo na fenda sináptica para atuar nos seus receptores, pós-sinápticos excitatórios e a risperidona é um antagonista desses receptores, como o 5HT2A, interferindo na ação da sertralina $(13,14,15,18)$.

O enalapril, uma pró-droga que por reações hidrolíticas é transformado em metabólito ativo; o enalaprilato (14), associado com risperidona, pode ocasionar em interação de potencialização e resultar em diminuição da pressão e hipotensão ortostática, uma RAM, pois a risperidona deprime a repolarização cardíaca que se reflete no intervalo QT, além de ser antagonista $\alpha 1$ e $\alpha 2$, promovendo o bloqueio da vasoconstrição mediada pelos receptores $\alpha$. Já o enalapril é indicado para reduzir a resistência vascular, através da inibição da enzima conversora de angiotensina $(4,13,15,17)$, como consequência o idoso apresentará tonturas e vertigens o que aumentará o risco de quedas e fraturas $(15,17)$.

Entre as interações medicamento-etanol encontram-se as interações com diazepam, clonazepam e risperidona, pois durante o período da pesquisa foi observado que o idoso A consumia álcool em horários pré-determinados, pois ele encontrava-se em terapia psicológica de redução de consumo, o que contempla o tratamento que preconiza a redução de danos (19), e estes horários eram próximos aos horários de administração dos fármacos citados.

O etanol é um modulador alostérico do receptor GABAA, que independe do GABA endógeno e aumenta o tempo de abertura do canal de cloreto. Sua associação com os BZD causa interação de potencialização de efeito depressor sobre o SNC, além de comprometer as funções psicomotora e motora; diminuir a atenção e pode aumentar a depressão respiratória (4).

O metabolismo do etanol ocorre por vias que envolvem oxidações, primeira à acetaldeído e segunda a ácido acético. O acetaldeído é um metabólito tóxico que pode causar a depleção da glutationa e ocasionar hepatotoxicidade (14). A interação do etanol com o paracetamol contribui para elevar o risco desta hepatotoxicidade
(17), pois na metabolização do paracetamol é gerado um metabolito tóxico, o N-acetil-p-benzoquinona- imina que deve ser inativado por conjugação com a glutationa, porém com a sua depleção (mediada pelo acetaldeído) há um acúmulo do metabólito tóxico que reage com constituintes nucleofílicos do hepatócito(14)

Uma associação benéfica, medicamento alimento, encontrada entre os fármacos utilizados pelo Idoso A, está no uso de ácido acetilsalicílico após o almoço, pois neste horário há redução do $\mathrm{pH}$ gástrico facilitando a absorção do fármaco $(4,15)$

O idoso B possui as seguintes patologias: Depressão parcial, insuficiência cardíaca congestiva (ICC), dislipidemia, fibrilação atrial e osteoporose. Foram encontradas 5 IM e duas destas ocasionaram em hipotensão, conforme descrito em evolução médica, levando ao surgimento de uma RAM. Em detalhe na tabela 2 os horários de administração e medicamentos de uso contínuo.

Entre as possíveis interações encontradas no idoso B destacam-se as interações de somação. O uso concomitante de enalapril e espironolactona é muito comum na prática clínica, principalmente como redutor de mortalidade na ICC (17).

Porém a associação do enalapril, inibidor da enzima conversora da angiotensina (ECA) e espironolactona, diurético poupador de potássio que age no ducto coletor, pode causar hipercalemia, mesmo quando administrado um diurético que causa depleção de potássio, como a furosemida, fármaco também utilizado pelo idoso. A inibição da ECA resulta na diminuição da secreção de aldosterona e da excreção de potássio, o que pode elevar os níveis do potássio sérico somado com a ação dos diuréticos poupadores de potássio $(13,17,18)$. Alguns sintomas de hiperpotassemia são: irritabilidade do miocárdio, arritmias, confusão mental, fraqueza dos músculos respiratórios e cardíacos (20).

Tabela 2: Medicamentos de uso contínuo do Idoso B

\begin{tabular}{|l|l|}
\hline Administração & \multicolumn{1}{|c|}{ Medicação } \\
\hline $6 \mathrm{~h} 00$ & omeprazol $40 \mathrm{mg}$ \\
\hline $8 \mathrm{~h} 00$ & carvedilol $25 \mathrm{mg}$; espironolactona $25 \mathrm{mg} ;$ \\
\hline $12 \mathrm{~h} 00$ & $\begin{array}{l}\text { enalapril } 10 \mathrm{mg} \text {; furosemida } 40 \mathrm{mg} ; \\
\text { isossorbida } 10 \mathrm{mg} \text {; dimeticona } 40 \mathrm{mg}\end{array}$ \\
\hline $14 \mathrm{~h} 00$ & carbonato de cálcio $500 \mathrm{mg}$ \\
\hline $16 \mathrm{~h} 00$ & furosemida $40 \mathrm{mg}$ \\
\hline $18 \mathrm{~h} 00$ & dimeticona $40 \mathrm{mg}$ \\
\hline $20 \mathrm{~h} 00$ & carvedilol $25 \mathrm{mg} ;$ varfarina $2,5 \mathrm{mg}$ \\
\hline $22 \mathrm{~h} 00$ & em dias alternados; sinvastatina $80 \mathrm{mg}$ \\
\hline
\end{tabular}

Fonte: Prescrições médicas do período de fevereiro de 2010 a fevereiro de 2011 
A associação do carvedilol, antagonista dos receptores $\alpha 1 ; \beta 1 ; \beta 2$, com furosemida, diurético de alça, ou com espironolactona também é muito frequente. Essa co-administração pode desencadear hiperglicemia e hipertrigliceridemia, e merece atenção, visto que o idoso já apresenta hiperlipidemia. Além disso, os diuréticos poupadores de potássio podem prolongar o intervalo QT e promover arritmias (17).

O uso da furosemida com enalapril promove uma interação de somação, todavia pode mediar uma interação de potencialização, pois o enalapril pode atenuar o aumento da excreção urinária de sódio causada pela furosemida, com isso a co-administração promove hipotensão e hipovolemia $(17,18)$.

Outra interação de potencialização verificada ocorre entre o enalapril e o mononitrato de isossorbida, que quando metabolizado libera óxido nítrico, este ativa os receptores guanilato ciclase citosólicos o que resulta em vasodilatação (15). O enalapril pode potencializar o efeito vasodilatador e hipotensor da isossorbida, além de reduzir a resistência vascular sistêmica e do trabalho cardíaco, aumentando ainda mais a eficácia da isossorbida (17).

Diante das interações apresentadas, evidenciou-se durante o período da pesquisa, que o idoso B apresentou uma reação adversa a medicamentos, ou seja, teve crises de hipotensão, sendo necessária a mudança de posologia de alguns fármacos, conforme descrito em evolução médica.

$\mathrm{Na}$ associação da varfarina; anticoagulante que atua bloqueando a epóxido redutase, que medeia a regeneração da vitamina $\mathrm{K}$ reduzida, com carvedilol e a sinvastatina; inibidora da enzima HMG CoA redutase, foi evidenciada interação farmacocinética que compreende a distribuição de fármacos $(13,18)$. A varfarina apresenta taxa de ligação à albumina de $99 \%$, portanto desloca os outros fármacos resultando em aumento da concentração plasmática livre e eleva a biodisponibilidade com intensificação das respostas farmacológicas, tanto terapêutica quanto tóxica $(4,13,15)$.

$\mathrm{O}$ idoso $\mathrm{C}$ apresenta hipotireoidismo, esteatose hepática, deficiência de vitamina B12, depressão, DPOC tabágico, gastrite atrófica e é etilista. Constatou-se 4 IM medicamento medicamento e 2 IM medicamento etanol. $\mathrm{Na}$ tabela $\mathrm{C}$ encontram-se horários de administração e as medicações de uso contínuo. A interação mais relevante no Idoso C ocorre com a administração de um medicamento, que tem em sua composição a combinação de $30 \mathrm{mg}$ de codeína e $500 \mathrm{mg}$ de paracetamol, associado à mais $500 \mathrm{mg}$ de paracetamol, pois ao observar os horários de administração destes fármacos, verifica-se uma dosagem excessiva de paracetamol. A dose diária segura do paracetamol é de $4 \mathrm{~g}$ ou de $2 \mathrm{~g}$ para usuários de etanol
(15) e este idoso utiliza 3,500g diário, logo as enzimas que catalisam as reações normais de conjugação podem estar saturadas, o que leva as oxidases de função mista converterem o paracetamol em um metabólito tóxico, o $\mathrm{N}$-acetil-p-benzoquinona- imina (14). A glutationa é responsável em inativar este metabólito, porém este idoso apresenta esteatose hepática, a qual leva a um comprometimento das funções hepáticas e por ter sido etilista crônico, sugere-se que há uma depleção dos níveis de glutationa, situação esta, que contribui para a presença do metabólito tóxico e consequente hepatotoxicidade (13).

As interações medicamento-etanol estão associadas com o uso do clonazepam, trazodona, codeína e resultam em interações de potencialização do efeito depressor do SNC (17). A associação do etanol e paracetamol resulta em hepatotoxicidade, pois o etanol é indutor enzimático e irá interferir na metabolização do paracetamol aumentado os níveis do N-acetil-p-benzoquinona- imina (14). Este idoso também encontra - se em terapia de redução de danos e têm horários pré-estabelecidos para consumir bebidas alcoólicas fora da instituição e estes horários são próximos aos da administração dos fármacos supracitados.

Tabela 3: Medicamentos de uso contínuo do Idoso C

\begin{tabular}{|c|l|}
\hline Administração & \multicolumn{1}{|c|}{ Medicação } \\
\hline 6 h00 & omeprazol 40mg; levotiroxina $75 \mathrm{mcg}$ \\
\hline 7 codeína $30 \mathrm{mg}+$ paracetamol $500 \mathrm{mg}$ \\
\hline $8 \mathrm{~h} 00$ & domperidona $1 \mathrm{mg}$ \\
\hline $11 \mathrm{~h} 00$ & venlafaxina $150 \mathrm{mg}$; paracetamol $500 \mathrm{mg}$ \\
\hline $12 \mathrm{~h} 00$ & domperidona $1 \mathrm{mg}$ \\
\hline $16 \mathrm{~h} 00$ & codeína $30 \mathrm{mg}+$ paracetamol $500 \mathrm{mg}$ \\
\hline $17 \mathrm{~h} 00$ & paracetamol $500 \mathrm{mg}$ \\
\hline $18 \mathrm{~h} 00$ & domperidona $1 \mathrm{mg}$ \\
\hline $20 \mathrm{~h} 00$ & codeína $30 \mathrm{mg}+$ paracetamol 500mg \\
\hline $22 \mathrm{~h} 00$ & trazodona $100 \mathrm{mg} ;$ clonazepam 0,25 mg \\
\hline & paracetamol $500 \mathrm{mg} ;$ \\
\hline
\end{tabular}

Fonte: Prescrições médicas do período de fevereiro de 2010 a fevereiro de 2011

A codeína, agonista do receptor $\mathrm{m}$ inibitório do plexo mioentérico, diminui a velocidade de esvaziamento gástrico e a motilidade intestinal $(4,14)$. Portanto é possível haver uma interação farmacocinética de absorção na combinação de codeína e paracetamol com levotiroxina.

A venlafaxina, inibidor de recaptação da serotonina e noradrenalina, é inibidor da enzima CYP3A4 que é responsável pela metabolização do paracetamol (15). Há de se considerar que a co-administração desses fármacos 
produz uma interação farmacocinética de biotransformação por mecanismo de inibição enzimática.

Verifica-se também uma interação farmacodinâmica de potencialização do clonazepam com a trazodona, antidepressivo atípico com ação hipnótica, aumentando os efeitos hipnóticos (18).

\section{CONCLUSÕES}

Ao analisar os fármacos prescritos aos 3 idosos, observou-se a presença de 17 possíveis interações medicamentosas. A maioria dessas interações pode comprometer a segurança e a eficácia terapêutica, logo diminuirá a efetividade do tratamento e a qualidade de vida do idoso. Portanto sugere-se buscar medidas preventivas que possibilitem a: substituição dos fármacos, redução de doses ou distanciamento dos horários de administração, através de soluções cabíveis que não acarretem custos ou recursos adicionais à instituição.

\section{AGRADECIMENTOS}

À Direção da Instituição de Longa permanência pelo consentimento em permitir a realização desta pesquisa.

Ao Professor Dr. Ricardo Wagner da UNIBRASIL e à Professora Dra Cristina Peitz de Lima da Universidade Federal do Paraná pelas sugestões

\section{REFERÊNCIAS}

1 Flores LM, Mengue SS. Uso de medicamentos por idosos em região do Sul do Brasil. Rev Saúde Pública. 2005; 39(6): 924-929.

2 Soares MA, Fernandez-Llimós F, Lança C, Cabrita J, Morais JA. Operacionalização para Portugal Critérios de Beers de Medicamentos Inapropriados nos Doentes Idosos. Acta Med Port. 2008; 21: $441-452$.

3 Rocha CH, Oliveira APS, Ferreira C, Faggiani FT, Schroeter G, Souza ACA, et al. Adesão à prescrição médica em idosos de Porto Alegre, RS. Ciência \& Saúde Coletiva. 2008; 13 (Sup): 703710 .

4 Oga S, Basile AC, Carvalho MF. Guia Zanini-Oga de interações medicamentosas: base teórica das interações. São Paulo: Atheneu; 2002

5 Secoli RS. Polifarmácia: interações e reações adversas no uso de medicamentos por idosos. Rev Bras Enferm. 2010; 63(1): 13640.

6 Karnikowski MGO, Nobrega OT. A terapia medicamentosa no idoso: cuidados na medicação. Ciência \& Saúde Coletiva. 2005; 10(2): 309-313

7 Novato ALS. Perfil de prescrição de medicamentos e interações medicamentosas em pacientes acima de 60 anos atendidos em hospital universitário: uma contribuição à farmacovigilância. São Paulo. Tese [ livre- docência]. Programa de Pós-Graduação em Fármaco e Medicamentos,Faculdade de Ciências Farmacêuticas, Universidade de São Paulo. 2005.

8 Correr CJ, Pontarolo R, Ferreira LC, Baptistão SAM. Riscos de problemas relacionados com medicamentos em pacientes de uma instituição geriátrica. Revista Brasileira de Ciências Farmacêuticas. Braz J Pharm Sciences. 2007; 43(1): jan./mar.

9 Andrade MA, Silva MVS, Freitas O. Assistência farmacêutica como estratégia para o uso racional de medicamentos em idosos. Semina cienc.biol.saúde. 2004; 25: 55-63.
10 Brito GC, Menezes MS, Mesquita AR, Lyra Junior DP. Efeito de um programa de manejo farmacoterapêutico em um grupo de idosos com hipertensão em Aracaju-Sergipe. Rev Ciênc Farma Básica Apl. 2009; 30(1): 83-89.

11 Novaes MRCG. Atenção farmacêutica ao idoso. Prática Hospitalar. 2007; Ano IX, N 52

12 Storpirtis S, Mori ALPM, Yochiy A, Ribeiro E, Porta V. Interações medicamentosas. In: Ciências Farmacêuticas Farmácia Clínica e Atenção Farmacêutica. Rio de Janeiro : Guanabara Koogan; 2008. p. 58-59.

13 Golan DE, Tashjian AHJ, Armstrong EJ, Armstrong AW. Princípios de farmacologia. A base fisiopatológica da farmacoterapia. $2^{\mathrm{a}}$ ed. Rio de Janeiro : Guanabara Koogan; 2009.

14 Rang HP, Dale MM, Ritter JM, Moore PK. Farmacologia. $5^{\text {a }}$ ed. Rio de Janeiro : Elsevier; 2004

15 Bruneton LL, Lazo JS, Parker KL. Goodman $\varepsilon$ Gilman. As bases farmacológicas da terapêutica. 10 ed. Rio de Janeiro: McGrawHill; 2007.

16 Fick DM, Cooper JW, Wade WE, Waller JL, Maclean JR, Beers MH. Updating the Beers Criteria for Potentially Inappropriate Medication Use in Older Adults . Results of a US Consensus Panel of Experts . Arch Intern ed. 2003;163: 2716-2724.

17 Drug information online. Acesso em 22/04/2011 Disponível em: http://www.drugs.com/drug interactions.php\#.

18 Grahame-Smith DG, Aronson JK. Tratado de farmacologia clinica e farmacoterapia. 3 ed. Rio de Janeiro : Guanabara Koogan; 2002.

19 Red Chilena reducción de dãnos. Acesso em 10/08/2011. Disponível em: .http://www.reducciondedanos.cl/wp/?page_id=14.

20 Motta VT. Bioquímica clínica: princípios e interpretações. Acesso em 10/05/2011. Disponível em: http://www.scribd.com/ doc/7194784/Bioq 